\title{
Event-Related fMRI Study of Response Inhibition
}

\author{
Peter F. Liddle, ${ }^{1,2 *}$ Kent A. Kiehl,, ${ }^{1,2}$ and Andra M. Smith ${ }^{1,2}$ \\ ${ }^{1}$ Department of Psychiatry, University of British Columbia, Vancouver, British Columbia, Canada \\ ${ }^{2}$ Department of Psychology, University of British Columbia, Vancouver, British Columbia, Canada
}

\begin{abstract}
Event-related functional magnetic resonance imaging (erfMRI) was employed to measure the hemodynamic response during a Go/No-go task in 16 healthy subjects. The task was designed so that Go and No-go events were equally probable, allowing an unbiased comparison of cerebral activity during these two types of trials. In accordance with prediction, anterior cingulate was active during both the Go and No-go trials, dorsolateral and ventrolateral prefrontal cortex was more active during the No-go trials, while primary motor cortex, supplementary motor area, pre-motor cortex and cerebellum were more active during Go trials. These findings are consistent with the hypothesis that the anterior cingulate cortex is principally engaged in making and monitoring of decisions, while dorsolateral and ventral lateral prefrontal sites play a specific role in response inhibition. Hum. Brain Mapping 12:100-109, 2001.

๑ 2001 Wiley-Liss, Inc.
\end{abstract}

Key words: response inhibition; frontal cortex; fMRI

\section{INTRODUCTION}

The ability to inhibit behavioral responses that are inappropriate in the current context is an essential component of normal behavior. It can be studied using Go/No-go tasks in which the participant is required to refrain from responding to designated items within a series of stimuli. Studies of the effects of lesions, and also studies using event-related potential (ERP) techniques, magnetoencephalography (MEG), and functional imaging studies using fMRI, provide evidence that neural circuits involving diverse areas of frontal cortex, and other association cortex sites such as parietal cortex, are implicated in the inhibition of response during No-go trials.

Lehmkuhler and Mesulam [1985] reported a patient with a medial prefrontal meningioma who performed poorly on Go/No-go tasks. The impairment resolved

*Correspondence to: P.F. Liddle, 2255 Wesbrook Mall, Vancouver, BC, V6T 2A1, Canada. E-mail: liddle@interchange.ubc.ca

Received for publication 5 October 1999; accepted 5 September 2000 when the tumour was resected. In an ERP study in which electrical potentials were recorded via subdural electrodes in five patients under investigation for epilepsy, Ikeda et al. [1996] identified transient electrical activity over the ventral (orbital) surface of the brain and also over the medial frontal cortex, peaking approximately $300 \mathrm{~ms}$ after the No-go stimulus. These potentials peaked before the electrical activity in the supplementary motor area, suggesting that they were associated with the decision whether or not to move. However, virtually identical potentials were recorded following Go trials, demonstrating that they did not reflect neural activity involved directly in suppression of the inappropriate response.

Studies using surgically implanted electrodes in monkeys [Sasaki et al., 1989] and employing MEG in human subjects [Sasaki et al., 1993] reveal activity in dorsolateral prefrontal cortex that is present following No-go trials, but not during Go trials. Sasaki et al. [1993] concluded that the dorsolateral prefrontal cortex is engaged in the actual suppression of inappropriate responses. 
Recent fMRI studies of regional cerebral activity during Go/No-go tasks have confirmed that diverse frontal loci play a role. Casey et al. [1997] found activation associated with response inhibition in orbital cortex, dorsal prefrontal cortex, and in anterior cingulate. In particular, they found a significant negative correlation between activity in the orbital frontal cortex and the number of errors of commission, suggesting that orbital under-activity predisposes to poor inhibition of responses. In a study of healthy controls and schizophrenic subjects, Rubia et al. [1998] reported that the healthy subjects exhibited increased activity associated with response inhibition in the anterior cingulate, and bilaterally in parietal cortex. The activation in medial frontal cortex was significantly reduced in schizophrenic subjects compared with the healthy subjects. Smith et al. [1998] found that the strongest activation during a Go/No-go task was in lateral frontal cortex. Furthermore, in a comparison between two types of Go/No-go epoch that differed in the degree of difficulty in suppressing responses during the No-go trials, Smith et al. found that the degree of activation of lateral frontal cortex was greater when the degree of difficulty in suppressing responses was greater.

The studies by Casey et al. [1997], Rubia et al. [1998], and Smith et al. [1998], all employed block designs that compared Go/No-go epochs, during which Go and No-go trials were presented, with baseline epochs during which there were no No-go trials. When employing block designs it is difficult to control for the difference in frequency of motor responses between blocks that differ in the proportion of Go and No-go events without creating differences in the rate of presentation of stimuli between blocks. Casey et al. [1997] and also Rubia et al. [1998] partially circumvented this difficulty by comparing the epochs containing Go and No-go responses with two baseline conditions that contained only Go trials: a baseline in which the frequency of Go trials matched that in Go/No-go epoch (ensuring approximate matching of number of motor responses) and a baseline in which the total number of trials matched Go/No-go epoch (ensuring matching of the number of stimuli presented). However, there is an even more serious limitation of block designs that compare with a baseline in which the trials are all Go trials: these Go trials are executed under conditions in there is very low demand upon the process of decision making. Therefore, differences between the Go/No-go epochs and the baseline epochs might reflect aspects of decision making or decision monitoring, rather than processes specifically related to response inhibition. The data presented by Casey et al. [1997] provide an indication that the activation of anterior cingulate might be related to monitoring errors. They found a strong positive correlation between the activation of anterior cingulate and the number of errors.

It is potentially more informative to employ event related designs in which the hemodynamic response to each type of event is recorded. With event related designs it is in principle possible to measure the hemodynamic response associated with Go trials and that with No-go trials under circumstances in which there is a decision to be made. It is also possible to distinguish the hemodynamic response associated with correct No-go trials from that associated with errors of commission during No-go trials. Using an event related design, Konishi et al. [1998] found that right infero-lateral prefrontal cortex was engaged specifically during No-go trials. In a later account of that study, they also reported less-significant activation in left inferior prefrontal cortex during No-go trials [Konishi et al., 1999]. However, Konishi and colleagues examined only four slices (each $7.5 \mathrm{~mm}$ thick) in a small sample of subjects. It is possible that this study failed to identify the full extent of involvement of frontal cortex due to the limited field of view and/or limited statistical power.

In an fMRI study using an event related design that was designed to induce a relatively high error rate, by virtue of presenting only a low proportion of No-go events, Kiehl et al. [2000] recently demonstrated that both lateral frontal cortex and anterior cingulate cortex were active during No-go trials [Kiehl et al., 2000]. However, in that study, Kiehl et al. also observed that the anterior cingulate cortex was active during Go trials. This observation supports the proposal by Carter et al. [1998] that the anterior cingulate is engaged during conditions that entail strong response competition. In addition, Kiehl et al. found that a more rostral part of the anterior cingulate, together with left lateral frontal cortex were active during errors of commission [Kiehl et al., 2000], raising the possibility that the anterior cingulate is also involved in monitoring responses.

A recent event-related fMRI study by Garavan et al. [1999] also reported activity in lateral frontal cortex and parietal cortex during No-go trials. However, in that study, the decision whether or not to make a response was based on comparison of the current stimulus with stimuli presented earlier in the series. The heavy demand this task places upon working memory makes it more difficult to interpret the observed pattern of cerebral activity.

While recent ERP and fMRI findings confirm the involvement of diverse loci within the frontal lobes, 
and also, parietal cortex, in the execution of Go/No-go tasks, they indicate that the different loci make differing contributions to the performance of the task. In particular, the evidence indicates that medial frontal cortex, including anterior cingulate, is engaged in decision formation and monitoring, while dorsolateral prefrontal cortex plays a specific role in response inhibition. The role of the ventral frontal cortex is less clear. However, when the results from Go/No-go studies are combined with evidence from other response inhibition tasks, it appears likely that ventral prefrontal cortex has a specific role in response inhibition. For example, Mishkin [1964] demonstrated that in monkeys, lesions of ventrolateral prefrontal cortex resulted in impaired ability to inhibit inappropriate responses.

In this paper, event-related fMRI techniques were used to distinguish between the cerebral activity associated with making the decision between responding or not responding, irrespective of whether or not the trial was a Go or a No-go trial, and the cerebral activity that differs between Go and No-go trials. A cardinal feature of the experimental design was creating a situation that allowed separate measurements of the hemodynamic response elicited by Go trials and by No-go trials presented under circumstances in which there was equal prior probability of a trial being a Go or a No-go trial.

In most Go/No-go tasks, the degree of difficulty of inhibiting responses in No-go trials is increased by presenting a preponderance of Go trials. One consequence of this is that the No-go trials necessarily entail the detection of a relatively rare stimulus. However, in the classic auditory 'oddball' paradigm, in which rare or novel stimuli are presented within a series of regular stimuli, these oddball stimuli elicit cerebral activity at a diverse range of cerebral sites, including orbital and lateral frontal cortex [Halgren et al., 1998; Kiehl et al., in press]. The activation is more extensive when a response to the rare stimuli is required, but nonetheless, occurs even when no motor response is required. Therefore, in a Go/No-go task in which the No-go events are rare, it is difficult to exclude the possibility that differences in cerebral activity between trial types are due the detection of 'oddball' stimuli rather than to inhibition. Presentation of equal numbers of Go and No-go trials reduces the likelihood that differences in cerebral activity between trial type can be attributed to the oddball character of the No-go stimuli.

In the task employed in this study, in which Go and No-go trials were equally probable, the degree of difficulty was enhanced by presenting the Go and No-go trials after a count down that heightened the readiness to respond rapidly when a stimulus was presented. Furthermore, a pulse sequence was employed that allowed coverage of the entire brain, including cerebellum.

In light of the evidence reviewed above indicating that anterior cingulate is principally engaged in making and monitoring the decision to respond, while dorsolateral and ventral (or orbital) cortex play a more specialized role in response inhibition during No-go trials, the following hypotheses were tested: 1) anterior cingulate will be active during both Go and No-go trials; 2) dorsolateral and ventral prefrontal cortex will be more active during No-go trials than during Go trials; and 3) motor areas including primary motor cortex, SMA, pre-motor cortex and cerebellum will be more active during Go trials than during No-go trials.

\section{METHODS}

\section{Participants}

Sixteen healthy right-handed participants with normal vision (nine men and seven women, mean age 30.2 years; SD 9.1) took part. Participants provided written informed consent and were screened to ensure that they satisfied MRI safety requirements. All procedures met with University and Hospital ethical approval.

\section{Task}

Stimuli were presented to the participant by a computer controlled projection system that delivered a visual stimulus to a rear-projection screen located at the entrance to the magnet bore. The participant viewed this screen through a system of mirrors attached to the top of the head coil. The distance between the subject's eyes and the screen was approximately six feet. The scanning room and magnet bore were darkened to allow easy visualization of the experimental stimuli. The stimulus for Go trials was the letter ' $X$ ' and the stimulus for No-go trials was 'A,' each presented within $62 \mathrm{~cm} \times 32 \mathrm{~cm}$ rectangular box for a period of $250 \mathrm{~ms}$. A single run containing $24 \mathrm{Go}$ and 24 No-go trials was presented.

The presentation of each trial began with a descending series of asterisks presented on the screen so as to heighten preparedness to respond. The series started with five asterisks presented for $250 \mathrm{~ms}$, followed by a blank screen $750 \mathrm{~ms}$; then four asterisks were presented for $250 \mathrm{~ms}$ followed by $750 \mathrm{~ms}$. The countdown continued until in this manner, ending with a single asterisk presented for $250 \mathrm{~ms}$, a blank screen for 750 $\mathrm{ms}$, and then either an ' $\mathrm{X}$ ' or an 'A' presented for 250 
ms, followed by a blank screen for $750 \mathrm{~ms}$. Between trials, a blank screen was presented for 3,4 , or $5 \mathrm{sec}$, so that the interval between the presentation of ' $X$ ' or ' $A$ ' stimuli was 9, 10, or $11 \mathrm{sec}$. The variation of this interval created variation in the relationship between the commencement of each pulse sequence repetition period and the presentation of stimuli, thereby ensuring that the hemodynamic response was sampled at 1-sec intervals.

Participants were instructed to respond as quickly and accurately as possible with their right index finger every time ' $X^{\prime}$ was presented and not to respond when 'A' was presented. A commercially available MRI compatible fiber-optic response device (Lightwave Medical, Vancouver, B.C.) was used to record the responses. Reaction times were computed on Go trials for which the participant responded within 1,200 ms post-stimulus. The absence of a response within a period of 1,200 ms following the onset of a Go stimulus was taken to be an error of omission. Responses within 1,200 ms after a No-go stimulus were regarded as errors of commission. Prior to entry into the scanning room, each participant performed a practice block of 10 trials to ensure understanding of the instructions.

\section{Imaging}

Imaging was performed using a clinical GE $1.5 \mathrm{~T}$ whole body MRI system fitted with a Horizon echospeed upgrade. The participant's head was firmly secured using a custom head holder and positioned approximately parallel to the anterior commissureposterior commissure (AC-PC) line using external references. Conventional spin echo $\mathrm{T}_{1}$ weighted sagittal localizers were acquired to confirm the location. Functional image volumes were collected with a gradientecho sequence (TR/TE 3,000/40 ms, flip angle $90^{\circ}$, FOV $24 \times 24 \mathrm{~cm}, 64 \times 64$ matrix, $62.5 \mathrm{kHz}$ bandwidth, 3.75 by $3.75 \mathrm{~mm}$ in plane resolution, $5 \mathrm{~mm}$ slice thickness, 29 slices) effectively covering the entire brain. A total of 167 images of the entire brain were collected in a total period of $501 \mathrm{sec}$. The presentation of stimuli did not commence until after $12 \mathrm{sec}$, to allow time for $\mathrm{T} 1$ effects to stabilize, and the four scans performed during this time were excluded from the analysis. In addition, scanning continued for $20 \mathrm{sec}$ after the final stimulus, to ensure that the hemodynamic response to that stimulus was adequately sampled.

\section{Image processing}

Functional images were reconstructed offline. For each subject, the scans were realigned and corrected for motion using the procedure of Friston et al. [1995] as implemented in Statistical Parametric Mapping (SPM97). Translation and rotation corrections did not exceed $1.0 \mathrm{~mm}$ and 1.5 degrees, respectively, for any of the participants. A mean functional image volume was constructed for each participant from the realigned image volumes. This mean image volume was then used to determine parameters for spatial normalization into the modified Talairach space employed in SPM97. These parameters were then applied to the corresponding functional image volumes for each participant. The normalized functional images were smoothed with an $8 \mathrm{~mm}$ full width at half-maximum Gaussian filter. Variations in global signal intensity were removed using proportional scaling. (In view of the fact that the issue of controlling for global variance in signal in fMRI studies is currently a matter of controversy and research, we also performed a supplementary analysis in which no global normalization procedure was employed.)

Low frequency noise was removed using a high pass filter (cutoff period $89 \mathrm{sec}$ ) [Holmes et al., 1997]. We also applied a notch filter at the Nyquist frequency (period of $6 \mathrm{sec}$ ) to remove noise associated with alternations of the applied radio frequency field. Eventrelated responses to the target and novel stimuli were modeled using a synthetic hemodynamic response function composed of two gamma functions, together with their temporal derivatives [as proposed by Josephs, Turner and Friston, 1997, and illustrated by Friston et al., 1998]. The first gamma function modeled the hemodynamic response using a peak latency of 6 sec. The second gamma function was used to model the small 'overshoot' of the hemodynamic response on recovery [Josephs et al., 1997]. Terms comprising the temporal derivatives of the gamma functions with multiplying factors that adjusted to give the optimum fit to the data, were included in the model to allow for variations in the peak latency.

There are several advantages of modeling the hemodynamic response in terms of basis functions, in this case, gamma functions and their derivatives. First, it has been shown that these gamma functions provide both reasonable and comprehensive models of the hemodynamic response [Boynton et al., 1996; Friston et al., 1994]. Second, by fitting the hemodynamic response for each voxel, we were able to effectively model variations in the hemodynamic response in both amplitude and latency between different brain regions (and between different events). Third, formulating the model in this way allows the use of standard procedures developed for analyzing serially correlated fMRI time-series that employ the general linear 
model and a sound theoretical correction for the number of comparison examined [Friston et al., 1995, Worsley and Friston, 1995].

\section{Statistical analysis}

A multisubject $(\mathrm{n}=16)$ statistical analysis was performed using the General Linear Model with a design matrix that included separate gamma functions and their respective temporal derivatives for the Go trials, the correct No-go trials, and the No-go error trials; a block effect; and the temporal filter, using SPM97. (There were no incorrect Go trials.) The resulting SPM (F) contained voxels in which the model fitted the observed data at a probability level of $P<0.001$. Four contrasts were then used to create $\operatorname{SPM}(\mathrm{t}) \mathrm{s}$ for our comparisons of interest. The four comparisons were: 1) Go trials vs. baseline; 2) correct No-go trials vs. baseline; 3) correct No-go vs. Go trials; 4) Go vs. correct No-go trials. The $\operatorname{SPM}(\mathrm{t}) \mathrm{s}$ were then transformed into SPM(Z)s. The No-go error trials were not entered into any contrasts because there were very few error trials, providing little power to estimate the hemodynamic response to these trials.

In determining the significance of $\mathrm{z}$ values, a correction for multiple comparisons based on the theory of Gaussian fields was employed [Worsley, 1994; Worsley and Friston, 1995]. Reported significance levels are at the voxel level [Worsley and Friston, 1995] and were all greater than $P<.05$ corrected for multiple comparisons unless otherwise noted.

It is important to note that there are three possible ways in which differences would be revealed in contrasts 3 and 4. First, activation for one event may be significantly greater than the activation for another event; second, one event may show activation and the other event may show deactivation; and third, one event may show deactivation but the other event may show significantly greater deactivation. In the present study there were no significant deactivations observed. Therefore, the observed differences between conditions are due to the presence of a significantly larger (e.g., more positive) response for one condition than the other.

\section{RESULTS}

\section{Behavioral data}

No subjects made errors of omission for Go trials. The mean reaction time for correct responses was 338 ms (SD $38 \mathrm{~ms}$ ). The mean number of errors of commission for No-go trials was $2.34 \%$ (SD 4.14). Four subjects made two errors of commission and one subject made one such error.

\section{Imaging data}

The sites of peak activation for the Go and No-go stimuli are listed in Tables I and II, respectively. During Go trials there was activation of anterior cingulate, supplementary motor area, contralateral motor cortex, bilateral parietal lobe and superior temporal gyrus, and cerebellum. During No-go trials activation was observed in bilateral parietal cortex, anterior cingulate cortex, and in dorsal prefrontal cortex. In the anterior cingulate cortex, activation during No-go trials was very similar to that during Go trials (see Fig. 1). The comparison of activation during No-go trials with that during Go trials revealed greater activation during No-go trials in bilateral inferior ventral and dorsal prefrontal cortex, bilateral parietal cortex and middle temporal cortex. The comparison of activation during Go trials with that during No-go trials revealed greater activation during Go trials in contralateral sensorimotor cortex, supplementary motor area, pre-motor cortex and ipsilateral cerebellum. These areas of activation for these latter comparisons are illustrated in Figure 2 and peak areas of activation are listed in Tables III and IV.

The supplementary analysis in which no global normalization procedure was employed yielded very similar results. All of the clusters of voxels in which there was a statistically significant activation for the Go or the No-go stimuli remained significant at the level $P<0.05$, in the absence of global normalization.

\section{DISCUSSION}

As predicted bilateral prefrontal cortex and anterior cingulate cortex were significantly active during the No-go trials. Furthermore, in accordance with hypotheses, the activation in anterior cingulate was similar during Go and No-go trials. This suggests that during No-go trials that anterior cingulate in involved in aspects of decision formation and monitoring that are similar for Go and No-go trials. In contrast, the dorsal and ventral lateral prefrontal cortex is more active during No-go trials than Go trials. The activity in dorso-lateral prefrontal cortex is consistent with the observation by Sasaki et al. [1993] using MEG, Casey et al. [1998] and Smith et al. [1998] employing fMRI with a block design, that lateral prefrontal cortex is specifically involved in response inhibition. The activity in ventrolateral prefrontal cortex is consistent with the observations of the effects of ventrolateral prefron- 
TABLE I. Summary of the significant areas of activation for the Go trials relative to the baseline condition ${ }^{\dagger}$

\begin{tabular}{|c|c|c|c|c|}
\hline \multirow[b]{2}{*}{ Region } & \multicolumn{3}{|c|}{$\begin{array}{l}\text { Talairach } \\
\text { coordinates }\end{array}$} & \multirow[b]{2}{*}{ z-score } \\
\hline & $x$ & $\mathrm{y}$ & $\mathrm{z}$ & \\
\hline \multicolumn{5}{|l|}{ Frontal lobes } \\
\hline 1. L Precentral Gyrus & -38 & -26 & 65 & $8.23^{* * *}$ \\
\hline 2. Medial Frontal Gyrus & -8 & -15 & 70 & $7.77^{* * *}$ \\
\hline $\begin{array}{l}\text { 3. Anterior Cingulate Gyrus } \\
\text { Parietal Lobes }\end{array}$ & 0 & 15 & 45 & $6.69^{* * *}$ \\
\hline 4. L Superior Parietal Lobule & -26 & -41 & 70 & $8.91^{* * *}$ \\
\hline 5. L Inferior Parietal Lobule & -45 & -45 & 60 & $8.47^{* * *}$ \\
\hline 6. L Precuneus & -8 & -52 & 70 & $7.23^{* * *}$ \\
\hline 7. L Inferior Parietal Lobule & -64 & -38 & 30 & $6.76^{* * *}$ \\
\hline 8. R Inferior Parietal Lobule & 68 & -30 & 30 & $6.21^{* * *}$ \\
\hline 9. R Superior Parietal Lobule & 8 & -64 & 65 & $6.04^{* * *}$ \\
\hline 10. R Precuneus & 8 & -75 & 55 & $5.48^{* *}$ \\
\hline $\begin{array}{l}\text { 11. R Inferior Parietal Lobule } \\
\text { Temporal Lobes } \\
\text { 12. L Superior Temporal }\end{array}$ & 56 & -45 & 50 & $5.09^{*}$ \\
\hline $\begin{array}{l}\text { Gyrus } \\
\text { 13. R Superior Temporal }\end{array}$ & -60 & 15 & -10 & $8.30^{* * *}$ \\
\hline $\begin{array}{l}\text { Gyrus } \\
\text { 14. L Superior Temporal }\end{array}$ & 54 & 22 & -15 & $8.09^{* * *}$ \\
\hline Gyrus & -60 & -19 & 10 & $7.75^{* * *}$ \\
\hline $\begin{array}{l}\text { 15. R Superior Temporal } \\
\text { Gyrus }\end{array}$ & 68 & -19 & 15 & $7.28^{* * *}$ \\
\hline 16. L Superior Temporal & & & & \\
\hline Gyrus & -68 & -30 & 25 & $6.54^{* * *}$ \\
\hline Deep Grey & & & & \\
\hline 17. L Thalamus & -1 & -8 & 10 & $7.28^{* * *}$ \\
\hline 18. R Thalamus & 11 & -4 & 15 & $7.21^{* * *}$ \\
\hline 19. R Caudate & 14 & -8 & 24 & $7.21^{* * *}$ \\
\hline 20. L Thalamus & -4 & -22 & 15 & $7.19^{* * *}$ \\
\hline 21. L Caudate & -14 & -4 & 24 & $7.06^{* *}$ \\
\hline 22. R Thalamus & 8 & -34 & 5 & $7.06^{* * *}$ \\
\hline 23. L Thalamus & -11 & -4 & 15 & $7.06^{* * *}$ \\
\hline 24. R Thalamus & 8 & 0 & 5 & $6.61^{* * *}$ \\
\hline Occipital Lobes & & & & \\
\hline 25. R Cuneus & 8 & -90 & 35 & $6.82^{* * *}$ \\
\hline 26. L Cuneas & -4 & -90 & 35 & $6.45^{* * *}$ \\
\hline Cerebellum & & & & \\
\hline 27. R Cerebellum & 26 & -52 & -25 & $8.23^{* * *}$ \\
\hline 28. R Cerebellum & 19 & -68 & -50 & $7.79^{* * *}$ \\
\hline 29. L Cerebellum & -34 & -49 & -50 & $7.58^{* * *}$ \\
\hline 30. L Cerebellum & -34 & -49 & -30 & $7.56^{* * *}$ \\
\hline 31. R Cerebellum & 26 & -45 & -50 & $7.56^{* * *}$ \\
\hline 32. L Cerebellum & -26 & -71 & -50 & $6.98^{* * *}$ \\
\hline
\end{tabular}

tal lesions on response inhibition in monkeys [Miskin, 1964]. These findings also confirm and extend the results of other event-related fMRI studies of response inhibition [Kiehl et al., 2000; Konishi et al., 1998, 1999].

In addition to activation of frontal cortex, bilateral activation was also observed in parietal cortex during the No-go trials, but not during the Go trials. Bilateral parietal activation during Go/No-go epochs had previously been reported by Rubia et al. [1998] and by Smith et al. [1998]. Involvement of parietal association cortex together with bilateral frontal lateral activation is consistent with existence of reciprocal connections between lateral frontal cortex and parietal cortex, that are documented well in primates [Pandya and Seltzer,

\section{TABLE II. Summary of the significant areas of activation for the correct No-go trials relative} to the baseline condition ${ }^{\ddagger}$

\begin{tabular}{llll}
\hline & \multicolumn{2}{c}{$\begin{array}{c}\text { Talairach } \\
\text { coordinates }\end{array}$} & \\
\cline { 2 - 3 } Region & $\mathrm{x} \quad \mathrm{y} \quad \mathrm{z}$ & z-score
\end{tabular}

Frontal lobes

1. R Superior Frontal Gyrus

2. Anterior Cingulate Gyrus

3. R Superior Frontal Gyrus

4. R Medial Frontal Gyrus

Parietal Lobes

5. R Inferior Parietal Lobule $\quad \begin{array}{llll}52 & -52 & 50 & 6.71^{* * *}\end{array}$

6. L Superior Parietal Lobule $\begin{array}{lllll}-26 & -41 & 70 & 6.34^{* * *}\end{array}$

7. L Paracentral Lobule $\quad-8 \quad-34 \quad 75 \quad 5.55^{* *}$

8. L Inferior Parietal Lobule $\quad-45 \quad-49 \quad 60 \quad 5.02^{* *}$

9. R Precuneus $\quad 8 \quad-75 \quad 55 \quad 4.94^{*}$

Temporal Lobes

10. R Superior Temporal

$$
\text { Gyrus }
$$

11. L Superior Temporal

Gyrus

12. L Superior Temporal

Gyrus

Deep Grey

13. L Thalamus

Occipital Lobes

14. R Cuneus

15. L Cuneas

Cerebellum

16. R Cerebellum

17. L Cerebellum

18. L Cerebellum

19. R Cerebellum

\begin{tabular}{rrrr}
52 & 22 & -15 & $7.87^{* * *}$ \\
-52 & 19 & -15 & $7.46^{* * *}$ \\
-64 & 0 & -5 & $4.70^{*}$ \\
& & & \\
-15 & -4 & 15 & $4.70^{*}$ \\
& & & \\
8 & -82 & 45 & $6.85^{* * *}$ \\
-4 & -90 & 35 & $5.89^{* * *}$ \\
& & & \\
45 & -49 & -30 & $7.43^{* * *}$ \\
-26 & -68 & -50 & $6.88^{* * *}$ \\
-38 & -64 & -25 & $6.69^{* * *}$ \\
15 & -79 & -45 & $6.42^{* * *}$ \\
\hline
\end{tabular}

‡ L, left; R, right.

$* P \leq .05$.

** $P \leq .01$.

${ }^{* * *} P \leq .001$ corrected for multiple comparisons. 

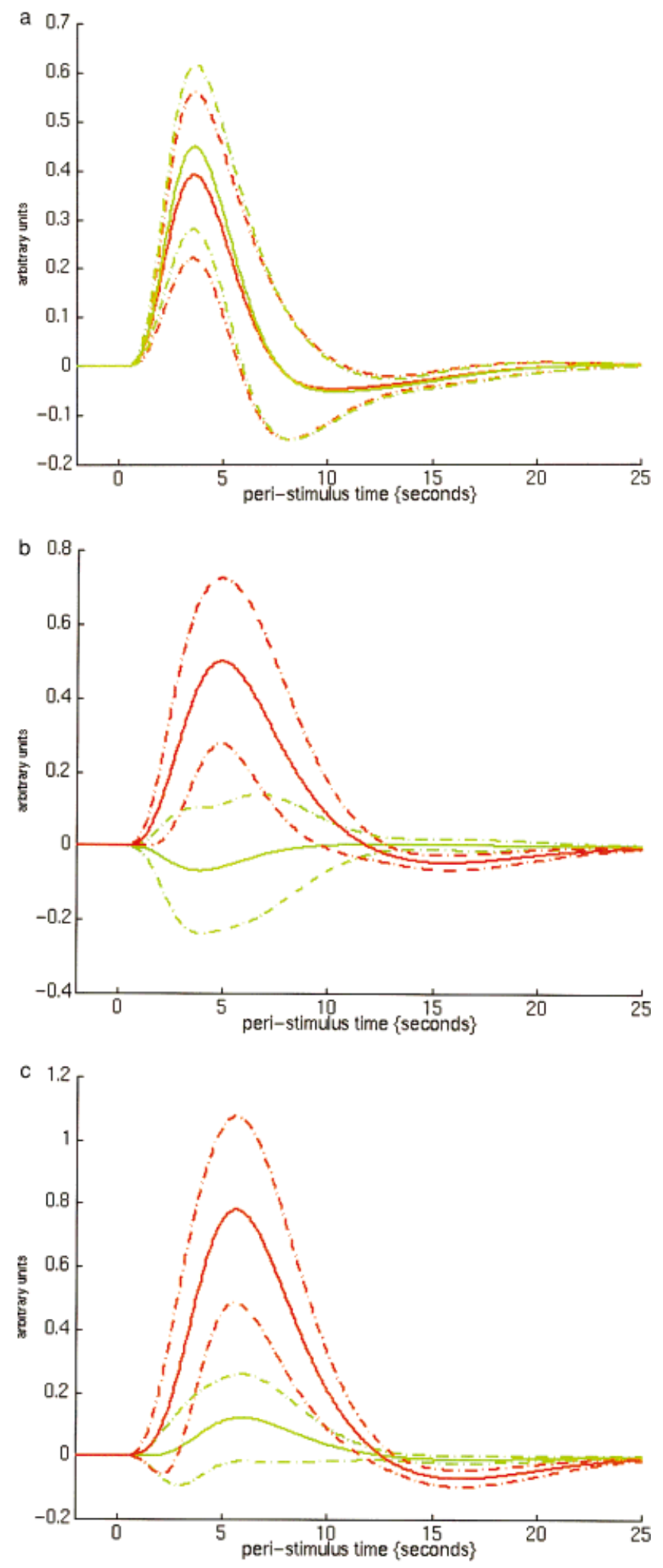

Figure I.
1982]. However, the nature of the contribution of parietal cortex to processing during No-go trials remains speculative.

In interpreting this study, it should be noted that the response to the count down procedure that preceded the events of interest might have contributed to the observed patterns of cerebral activity. The rise times of the fitted hemodynamic responses shown in Figure 1 are typical of responses to events localized at the time of the events of interest, and suggest that the major contribution to the responses that fitted the model were related directly to the Go or No-go stimuli rather than preceding events. Nonetheless, the possibility that the preceding count down made an appreciable contribution to the patterns of cerebral activity associated with each type of event cannot be excluded. This must be borne in mind when examining the patterns of cerebral activity associated with each specific trial type. However, because an identical count down preceded both Go trials and No-go trials, it is unlikely that the count down procedure could account for observed differences between responses to the Go and No-go trials.

In summary, lateral frontal cortex, and anterior cingulate cortex and parietal cortex are active during No-go trials in a Go/No-go task. However, the activation of anterior cingulate during No-go trials is not substantially greater than that during Go trials under circumstances in which No-go trials and Go trials are equally probable, in accord with the hypothesis that the activation of anterior cingulate is related to decision formation and monitoring, rather than to response inhibition. On the other hand, dorsal and ventral lateral prefrontal cortex are more active during No-go trials, indicating a specific role in response inhibition. In particular, at the loci in right and left lateral frontal cortex at which activation was most

Figure I.

Illustration of the hemodynamic response at three sites of interest: (a) anterior cingulate; (b) left ventro-lateral frontal cortex; and (c) right ventro-lateral cortex for Go trials (green) and No-go trials (red). For each type of trial, the mean fitted hemodynamic response is represented by the solid line. The upper dashed line represents $(Y+S . E$.$) and the lower dashed line represents (Y-$ S.E.) where $Y$ is the fitted response and S.E. is the standard error of the response. In anterior cingulate, the hemodynamic response at the site of peak activation for each trial type is shown. The peak activation in the Go trials is located at $(0,15,45)$ while the peak activation for No-go trials is located at $(4,19,45)$. In left and right ventro-lateral frontal cortex, the hemodynamic response to both types of trials is shown at the sites of most significant difference between the No-go and Go trials ( $-49,30,-15$ on the left; 4I,34, -15 on the right). 


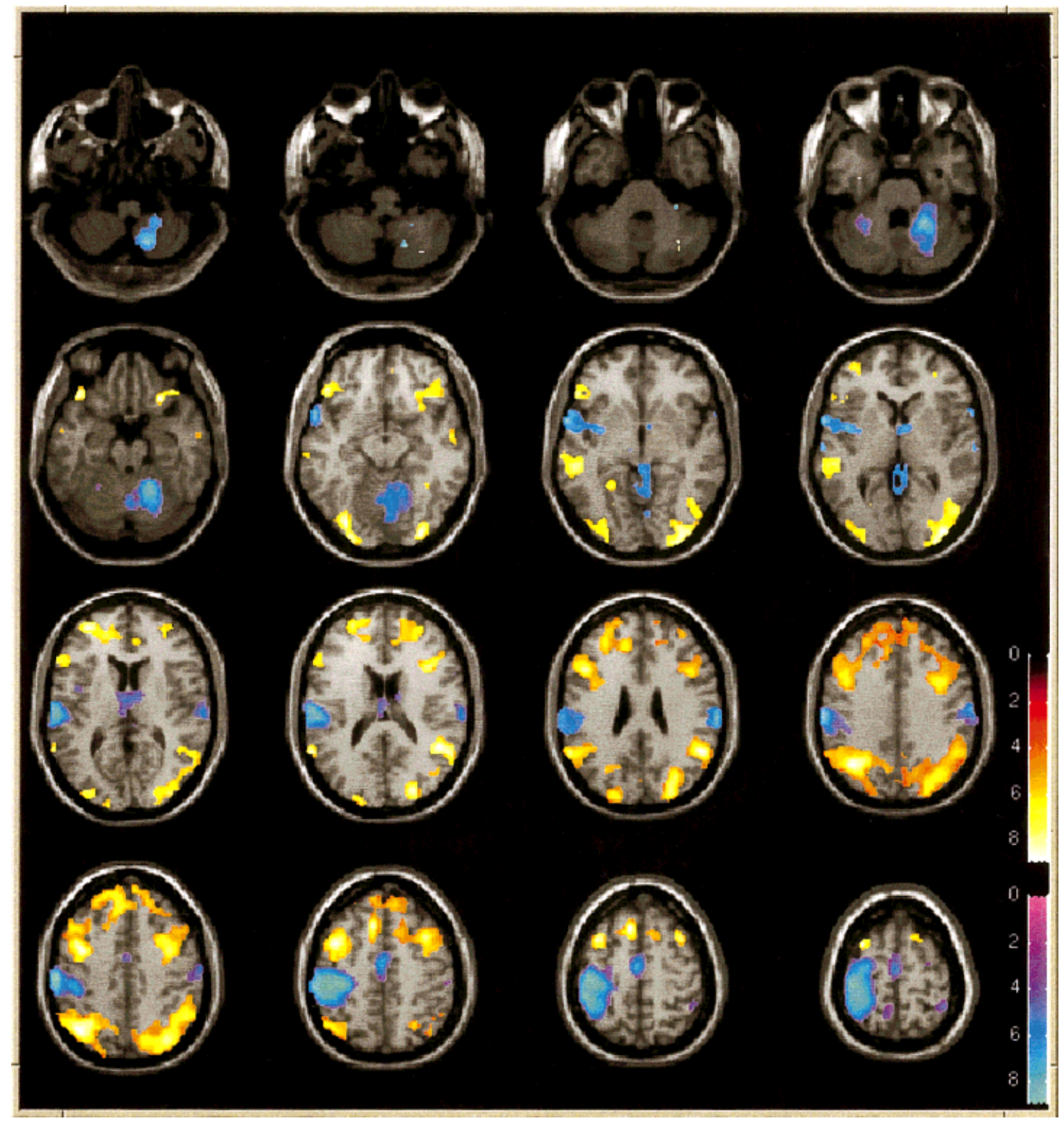

Figure 2.

Comparison of cerebral activity during No-go trials with that shown. The areas of activation are indicated on 16 axial slices 8 during Go trials. Sites of significantly greater activation during No-go trials compared with Go trials are shown in shades of orange. Sites of significantly greater activation during Go trials compared with No-go trials are shown in shades of blue. Only voxels in which the difference in activation was significant at the level $P<0.05$ after correction for multiple comparisons are $\mathrm{mm}$ apart. The lowest slice (shown on upper left) is at $-52 \mathrm{~mm}$ below the inter-commissural plane, while the highest slice (lower right) is at $68 \mathrm{~mm}$ above the inter-commissural plane. The slices are displayed according to neurological convention with the left hemisphere on the left.

significant during No-go trials, there was no substantial activation during Go trials. Although this study provided no evidence of significant activation of lateral prefrontal trials cortex during Go trials, the possibility that regions within the lateral prefrontal cortex

are engaged, at least to some extent, during Go trials cannot be excluded. Likewise, the absence of significant differences in activation between Go and No-go trials in the anterior cingulate cortex, does not rule out the possibility that there are loci within the anterior 
cingulate that differ in their engagement during Go and No-go trials. Nonetheless, our findings indicate a degree of dissociation between the roles of the lateral frontal cortex and anterior cingulate cortex during a Go/No-go task.

\section{ACKNOWLEDGMENTS}

We thank Drs. Alex MacKay, Bruce Forster, and Ken Whittall for their assistance. We also thank MR technicians Trudy Shaw, Karen Smith, and Sylvia Ren-

TABLE III. Summary of the significant areas of where the correct No-go trials produced a significantly larger hemodynamic response than did Go trials ${ }^{\ddagger}$

\begin{tabular}{llllll}
\hline & & \multicolumn{3}{c}{$\begin{array}{c}\text { Talairach } \\
\text { coordinates }\end{array}$} \\
\cline { 2 - 4 } Region & $\mathrm{x}$ & $\mathrm{y}$ & $\mathrm{z}$ & $\mathrm{z}$ \\
\hline
\end{tabular}

Frontal lobes

1. L Middle Frontal Gyrus

2. L Superior Frontal Gyrus

3. R Middle/Inferior Frontal Gyrus

4. L Middle/Inferior Frontal Gyrus

5. L Middle Frontal Gyrus

6. R Middle/Inferior Frontal Gyrus

7. L Middle/Inferior Frontal Gyrus

8. L Superior Frontal Gyrus

9. L Superior Frontal Gyrus

Parietal Lobes

10. L Inferior Parietal Lobule

11. R Inferior Parietal Lobule

12. L Supramarginal Gyrus

13. R Inferior Parietal Lobule

14. L Precuneas

Temporal Lobes

15. R Superior Temporal Gyrus

16. R Middle Temporal Gyrus

17. L Middle Temporal Gyrus

Occipital Lobes

18. R Cuneus

19. R Superior Occipital Gyrus

20. R Middle Occipital Gyrus

21. L Cuneus

Cerebellum

22. R Cerebellum

23. L Cerebellum

\begin{tabular}{|c|c|c|c|}
\hline-38 & 0 & 50 & $6.88^{* * * *}$ \\
\hline-8 & 22 & 55 & $6.27^{* * * *}$ \\
\hline 38 & 8 & 40 & $6.15^{* * * *}$ \\
\hline-45 & 19 & 30 & $5.92^{* * *}$ \\
\hline-34 & 52 & 10 & $5.49^{* *}$ \\
\hline 41 & 34 & -15 & $5.49^{* *}$ \\
\hline-49 & 30 & -15 & $5.26^{* *}$ \\
\hline-8 & 45 & 50 & $5.22^{* *}$ \\
\hline-30 & 40 & 34 & $4.83^{*}$ \\
\hline-38 & -64 & 40 & $6.92^{* * *}$ \\
\hline 30 & -79 & 40 & $5.83^{* * * *}$ \\
\hline-60 & -52 & 30 & $5.28^{* * *}$ \\
\hline 41 & -56 & 45 & $5.03^{*}$ \\
\hline-8 & -68 & 40 & $4.77^{*}$ \\
\hline 52 & -52 & 30 & $6.37^{* * *}$ \\
\hline 45 & -68 & 35 & $4.69^{*}$ \\
\hline-52 & -30 & 0 & $4.69 *$ \\
\hline 26 & -86 & 35 & $5.83^{* * *}$ \\
\hline 30 & -75 & 30 & $5.69^{* * *}$ \\
\hline 34 & -98 & 0 & $5.14^{*}$ \\
\hline-19 & -94 & 30 & $5.13^{* *}$ \\
\hline 38 & -94 & -10 & $6.09 *$ \\
\hline-26 & -94 & -10 & $4.89^{*}$ \\
\hline
\end{tabular}

$\ddagger$ L, left; R, right.

$* P \leq .05$.

** $P \leq .01$.

${ }^{* * *} P \leq .001$ corrected for multiple comparisons. neberg. K.A.K. was supported by the Michael Smith Graduate Studentship, and A.M.S. was supported by a Killam Graduate Scholarship.

\section{REFERENCES}

Blumer D, Benson DF (1975): Personality changes with frontal lobe lesions. In: Blumer D, Benson DF, editors. Psychiatric aspects of neurological disease. New York: Grune and Stratton p 151-170.

Boynton G, Engel S, Glover G, Heeger B (1996): Linear systems analysis of functional magnetic resonance imaging in human V1. J Neurosci 16:4207-4221.

Casey BJ, Trainor RJ, Orendi JL, Schubert, AB, Nystrom LE, Giedd JN, Haxby JV, Noll DC, Cohen JD, Forman SD, Dahl RE, Rapoport JL (1997): A developmental functional MRI study of prefrontal activation during performance of a Go-No-Go task. J Cogn Neurosci 9:835-847.

TABLE IV. Summary of the significant areas of where the Go stimuli produced a significantly larger hemodynamic response than did correct No-go trials

\begin{tabular}{|c|c|}
\hline & \\
\hline Region & $x$ \\
\hline
\end{tabular}

Frontal lobes

1. L Precentral Gyrus

2. L Postcentral Gyrus

$$
-38-26
$$

$658.71^{* * * *}$

3. L Medial Frontal Gyrus

$-30-41$

$708.40^{* * *}$

4. R Postcentral Gyrus

5. R Precentral Gyrus

6. L Insula

7. R Precentral Gyrus

Parietal Lobes

8. L Inferior Parietal Lobule

9. R Inferior Parietal Lobule

$\begin{array}{ll}0 & -11\end{array}$

$68-22$

$64-15$

$-41 \quad 0$

$64 \quad 15$

60

$256.69^{* * * *}$

$405.88^{* * *}$

Temporal Lobes

10. L Superior Temporal

Gyrus

11. L Transverse Temporal Gyrus

$\begin{array}{rrrr}-45 & -41 & 60 & 8.23^{* * *} \\ 41 & -45 & 65 & 4.99^{* *}\end{array}$

12. R Superior Temporal

Gyrus

Deep Grey

13. R Thalamus

Cerebellum

14. R Cerebellum

15. R Cerebellum

16. L Cerebellum

\begin{tabular}{rrrl}
-60 & 11 & -10 & $7.29^{* * *}$ \\
-60 & -19 & 15 & $7.50^{* * *}$ \\
68 & -19 & 10 & $5.89^{* * *}$ \\
& & & \\
0 & -8 & 15 & $5.22^{*}$ \\
26 & -49 & -25 & $8.00^{* * *}$ \\
19 & -64 & -50 & $6.59^{* * *}$ \\
-26 & -49 & -25 & $5.69^{* *}$ \\
\hline
\end{tabular}

${ }^{\ddagger} \mathrm{L}$, left; R, right.

$* P \leq .05$.

** $P \leq .01$.

${ }^{* * *} P \leq .001$ corrected for multiple comparisons. 
Carter CS, Braver TS, Barch DM, Botvinick MM, Noll DC, Cohen JD (1998): Anterior cingulate cortex, error detection, and the online monitoring of performance. Science 280:747-749.

Friston Jezzard P, Turner R (1994): Analysis of functional MRI time series. Human Brain Mapp 1:153-171.

Friston KJ, Fletcher P, Josephs O, Holmes A, Rugg MD, Turner R (1998): Event related fMRI: characterizing differential responses. NeuroImage 7:30-40.

Friston KJ, Holmes AP, Worsley KJ, Poline J-P, Frith CD, Frackowiak RSJ (1995): Statistical parametric maps in functional imaging; a general linear approach. Human Brain Mapp 2: 189-210.

Halgren E, Marinkovic K, Chauvel P (1998): Generators of the late cognitive potentials in auditory and visual oddball tasks. Electroencephalography Clin Neurophysiol 106:156-164.

Holmes AP, Josephs O, Buchel C, Friston KJ (1997): Statistical modelling of low frequency confounds in fMRI. Neuroimage 5:S480.

Ikeda A, Luders HO, Collura TF, Burgess RC, Morris HH, Hamano T, Shibasaki (1996): Subdural potentials at orbitofrontal and mesial prefrontal areas accompanying anticipation and decision making in humans: a comparison with Bereitschaftspotential. Electroencephalography Clin Neurophysiol 98:206-212.

Josephs O, Turner R, Friston K (1997): Event-related fMRI. Human Brain Mapp 5:1-7.

Kiehl KA, Liddle PF, Hopfinger JB (2000): Error processing and the rostral anterior cingulate: an event-related fMRI study. Psychophysiology 37:216-223.

Kiehl KA, Laurens, Duty TL, Forster BB, Liddle PF (in press): Neural sources involved in auditory target detection and novelty processing: an event-related fMRI study. Psychophysiology
Konishi S, Nakajima K, Uchida I, Sekihara K, Miyashita Y (1998): No-go dominant brain activity in human inferior prefrontal cortex revealed by functional magnetic resonance imaging. Euro J Neurosci 10:1209-1213.

Konishi S, Nakajima K, Uchida, I, Kikyo H, Kameyama M, Miyashita Y (1999): Common inhibitory mechanism in human inferior prefrontal cortex revealed by event-related functional MRI. Brain 122:981-991.

Mishkin M (1964): Perseveration of central sets after frontal lesions in monkeys. In: Warren JM, Akert K, editors. The frontal granular cortex and behavior. NewYork: McGraw-Hill. p 219-241.

Pandya DN, Seltzer B (1982): Intrinsic connections and architectonics of the posterior parietal cortex in the rhesus monkey. J Comp Neurol 204:196-210.

Rubia K, Russel T, Taylor E (1998): Brain activation in schizophrenia during performance of a Go-no-go task in fMRI. Schizophrenia Res 29:112-113.

Sasaki K, Gemba H (1986): Electrical activity in the prefrontal cortex specific to No-go reaction of conditioned hand movement with colour discrimination in the monkey. Exp Brain Res 64:603-606.

Sasaki K, Gemba H, Nambu A, Matsuzaki R (1993): No-go activity in the frontal association cortex of human subjects. Neurosci Res 18:249-252.

Smith AM, Kiehl KA, Mendrek A, Forster BB, Hare RD, Liddle PF (1998): Whole brain fMRI of a Go/No-go task. Neuroimage 7:S971-S971.

Worsley KJ, Friston KJ (1995): Analysis of fMRI time-series revisited-Again. Neuroimage 2:173-181.

Worsley KJ (1994): Local maxima and the expected Euler characteristic of excursion sets of chi2, F and t fields. Adv Appl Probability 26:13-42. 